\title{
简易合成 $\mathrm{Bi} / \mathrm{Bi}_{2} \mathrm{MoO}_{6} / \mathrm{TiO}_{2}$ 复合纳米纤维及其 增强的可见光催化性能
}

\author{
李晓萍 ${ }^{1}$, 李跃军 ${ }^{1}$, 曹铁平 ${ }^{1}$, 孙大伟 ${ }^{1,2}$, 王 霞 ${ }^{1}$, 席啸 天 ${ }^{1}$ \\ (1. 白城师范学院 化学学院, 白城 137000; 2. 吉林师范大学 化学学院, 四平 136000)
}

摘 要: 以电纺 $\mathrm{TiO}_{2}$ 纳米纤维为基质, 葡萄糖为还原剂, 采用简单一步溶剂热法制备了等离子体 $\mathrm{Bi} / \mathrm{Bi}_{2} \mathrm{MoO}_{6} / \mathrm{TiO}_{2}$ 复合纳米纤维。利用 $X$ 射线衍射、场发射扫描电镜、透射电子显微镜、 $X$ 射线光电子能谱、紫外-可见漫反射光谱 和光致发光谱等对样品进行表征。以 $\mathrm{RhB}$ 和 4-CP 为模拟有机污染物, 评价材料的光催化性能。结果表明: 部分 $\mathrm{Bi}^{3+}$ 被葡萄糖还原成金属 $\mathrm{Bi}$ 纳米粒子, 原位沉积在 $\mathrm{Bi}_{2} \mathrm{MoO}_{6}$ 纳米片上, 同时构筑在 $\mathrm{TiO}_{2}$ 纳米纤维表面。金属 $\mathrm{Bi}$ 的 等离子体共振效应, 有效提高了样品的光催化活性。可见光照 $50 \mathrm{~min}$, 样品对 $\mathrm{RhB}$ 的降解率为 $95.8 \%$, 五次循环后 仍保持在 $92 \%$ 以上; 可见光照 $180 \mathrm{~min}$, 样品对 4-CP 的降解率达 $68.8 \%$ 。证实该材料具有良好的可见光催化活性和 稳定性。

关 键 词: 金属 $\mathrm{Bi}$; 等离子体共振效应; 复合纳米纤维; 可见光催化

中图分类号: O643 文献标识码: A

\section{Facile Synthesis of $\mathrm{Bi} / \mathrm{Bi}_{2} \mathrm{MoO}_{6} / \mathrm{TiO}_{2}$ Composite Nanofibers with Enhanced Photocatalytic Activity under Visible Light}

\author{
LI Xiao-Ping ${ }^{1}$, LI Yue-Jun ${ }^{1}$, CAO Tie-Ping ${ }^{1}$, SUN Da-Wei ${ }^{1,2}$, WANG Xia ${ }^{1}$, XI Xiao-Tian ${ }^{1}$ \\ (1. College of Chemistry, Baicheng Normal University, Baicheng 137000, China; 2. College of Chemistry, Jilin Normal Uni- \\ versity, Siping 136000, China)
}

\begin{abstract}
The plasma $\mathrm{Bi} / \mathrm{Bi}_{2} \mathrm{MoO}_{6} / \mathrm{TiO}_{2}$ composite nanofibers were prepared via a facile one-step solvothermal method, using electrospun $\mathrm{TiO}_{2}$ nanofibers as substrate, and glucose as reducing agent. The photocatalytic activity of the samples were evaluated by photodegradation of rhodamine B and 4-chlorophenol solution under visible light irradiation. The results showed that metal $\mathrm{Bi}$ nanoparticles were generated on the surface of $\mathrm{Bi}_{2} \mathrm{MoO}_{6}$ nanosheets via reduction of $\mathrm{Bi}^{3+}$ in situ by glucose, meanwhile grew on the $\mathrm{TiO}_{2}$ nanofibers surface. The photocatalytic activity of the $\mathrm{Bi} / \mathrm{Bi}_{2} \mathrm{MoO}_{6} / \mathrm{TiO}_{2}$ composites nanofibers can be further improved by depositing metallic Bi owing to its surface plasmon resonance. The RhB catalyzed by the sample was degraded by $95.8 \%$ under visible light irradiation for $50 \mathrm{~min}$, and the degradation efficiency remained over $92 \%$ after 5 cycles while the 4-CP was degraded for $68.8 \%$ under visible light irradiation for $180 \mathrm{~min}$. All above results suggest that the photocatalysts have good photocatalytic activity and stability.
\end{abstract}

Key words: metal Bi; plasma resonance effect; composite nanofibers; visible light photcatalysis

收稿日期: 2018-12-03; 收到修改稿日期: 2019-03-10

基金项目: 国家自然科学基金(21573003); 国家级大学生创新创业训练计划项目(201810206003)

National Natural Science Foundation of China (21573003); National College Students' Innovative Entrepreneurship Training Program (201810206003)

作者简介: 李晓萍(1963-)，女，硕士，教授.E-mail: bclixp63@163.com

通讯作者: 李跃军, 教授. E-mail: bc640628@163.com 
半导体光催化氧化是最有前途的环境修复技术 之一。 $\mathrm{TiO}_{2}$ 具有廉价、无毒、环境友好和可重复利 用等诸多优点, 在光催化领域成为研究热点 ${ }^{[1-2]}$ 。但 是纯 $\mathrm{TiO}_{2}$ 带隙较宽, 只能吸收太阳光中不足 $5 \%$ 的 紫外光; 自身光生电子-空穴对易复合, 又导致光量 子效率较低，限制了其实际应用 ${ }^{[3-4]}$ 。

铋基光催化材料以其独特的能带结构和较高的 光腐蚀稳定性而引起了广泛关注 ${ }^{[5]}$ 。其中 $\mathrm{Bi}_{2} \mathrm{MoO}_{6}$ 性能稳定、带隙较窄 $(2.5 \sim 2.8 \mathrm{eV})$ 、形貌可控, 已经 制备出纳米片 ${ }^{[6]}$ 、纳米球 ${ }^{[7]}$ 、纳米纤维 ${ }^{[8]}$ 、纳米墙 ${ }^{[9]}$ 、 纳米花 ${ }^{[10]}$ 和纳米管 ${ }^{[11]}$ 等不同形貌的 $\mathrm{Bi}_{2} \mathrm{MoO}_{6}$ 光催 化剂, 并证实其光催化性能与材料的形貌和电荷分 离效率密切相关。但纯 $\mathrm{Bi}_{2} \mathrm{MoO}_{6}$ 光吸收效率低、电 荷转移速率慢和光致电荷载流子复合几率高, 致使 其光催化性能仍不能满足实际应用需求 ${ }^{[12]}$ 。

自 Awazu 等 ${ }^{[13]}$ 首次提出贵金属表面等离子体光 催化, 并成功将金属 $\mathrm{Au}$ 纳米粒子应用于开发具有可 见光驱动的光催化材料以来, $\mathrm{Au} 、 \mathrm{Ag} 、 \mathrm{Pt}$ 等贵金属光 催化引起人们广泛的研究兴趣 ${ }^{[14-17]}$ 。但是这些光催化 剂价格昂贵成为大规模推广应用的瓶颈，而半金属 $\mathrm{Bi}$ 具有成本低、带隙小、能带重叠少等优点, 特别是当 $\mathrm{Bi}$ 颗粒小于几十纳米时, 能够产生与贵金属相似的表 面等离子体共振(SPR)效应 ${ }^{[18-19]}$, 成为贵金属表面等 离子体光催化最理想的替代品。近年来, 有关研究者 将金属 $\mathrm{Bi}$ 与 $\mathrm{Bi}_{2} \mathrm{O}_{3} 、 \mathrm{TiO}_{2} 、(\mathrm{BiO})_{2} \mathrm{CO}_{3} 、 \mathrm{BiOCl}$ 和 $\mathrm{Bi}_{x} \mathrm{Br}_{1-x}$ 等半导体相复合制备了具有较高活性的可见光催化剂, 应用于降解有机污染物, 已取得了良好效果 ${ }^{[20-22]}$ 。本工 作采用一步溶剂热法合成了等离子体 $\mathrm{Bi} / \mathrm{Bi}_{2} \mathrm{MoO}_{6} / \mathrm{TiO}_{2}$ 复合纳米纤维光催化材料, 通过 $\mathrm{RhB}$ 和 4-CP 考察其 可见光催化活性及增强光催化机理。

\section{1 实验方法}

\section{1 试剂和仪器}

聚乙烯吡咯烷酮(PVP, Ms=1300000), 化学纯, 北 京益利精细化学品有限公司; 钛酸四丁酯 $\left(\mathrm{Ti}(\mathrm{OBu})_{4}\right)$, 分析纯, 上海昆行化工科技有限公司; 硝酸铋 $\left(\mathrm{Bi}\left(\mathrm{NO}_{3}\right)_{3} \cdot 5 \mathrm{H}_{2} \mathrm{O}\right)$ 、钿酸钠 $\left(\mathrm{Na}_{2} \mathrm{MoO}_{4} \cdot 2 \mathrm{H}_{2} \mathrm{O}\right)$ 和葡萄糖 $\left(\mathrm{C}_{6} \mathrm{H}_{12} \mathrm{O}_{6}\right)$, 分析纯, 国药集团化学试剂有限公司; 乙二醇、无水乙醇和冰醋酸, 分析纯, 北京化工厂; 二次蒸馏水(自制)。

利用 PANalytical $\chi^{\prime}$ Pert $^{3}$ Powder X 射线粉末衍 射仪(XRD)测量样品的物相, 采用 $\mathrm{CuK \alpha}$ 靶 $(\lambda=$ $0.154056 \mathrm{~nm})$, 管流 $40 \mathrm{~mA}$, 管压 $40 \mathrm{kV}$, 扫描范围
为 $20^{\circ} \sim 80^{\circ}$; 利用 Hitachi SU8010 型场发射扫描电子 显微镜(SEM)观测样品形貌, 工作电压为 $5 \mathrm{kV}$; 采 用 JEOL-JSM2010 型高分辨透射电子显微镜 (HRTEM) 观察样品显微结构, 工作电压为 $100 \mathrm{kV}$; 采用 PHI-5000 VersaProbe X 射线光电子能谱(XPS) 检测样品化学组成和元素价态, 以污染碳 $\mathrm{C} 1 \mathrm{~s}$ 峰 $(284.6 \mathrm{eV})$ 校正荷电位移; 采用 Lambda 35 型紫外可 见分光光度计 (PerkinElmer) 测定样品的紫外-可见 漫反射光谱(UV-Vis DRS), 以 $\mathrm{BaSO}_{4}$ 作为标准反射 率; 采用 Hitachi F-4500 荧光分光度计(激发波长为 $350 \mathrm{~nm}$ ) 进行苂光(PL)光谱分析。

\section{2 样品的制备}

$\mathrm{TiO}_{2}$ 纳米纤维的制备: 取 $1.0 \mathrm{~g}$ PVP 加入到 $10 \mathrm{~mL}$ 无水乙醇中, 磁力搅拌 $2 \mathrm{~h}$, 制得高分子溶液。取 $1.5 \mathrm{~mL}$ $\mathrm{Ti}(\mathrm{OBu})_{4}$ 溶于 $6 \mathrm{~mL}$ 无水乙醇与冰醋酸的混合溶液 $(V$ 乙䤃: $V$ 冰酔酸 $=1: 1)$, 持续搅拌 $30 \mathrm{~min}$, 缓慢滴加到上 述高分子溶液中, 强力搅拌 $2 \mathrm{~h}$, 再陈化 $2 \mathrm{~h}$ 后转移 至电纺用注射器。 $15 \mathrm{kV}$ 电压, 接收距离 $15 \mathrm{~cm}$, 电 纺 $4 \mathrm{~h}$, 取下纤维毡真空干燥 $12 \mathrm{~h}$ 后, 置于马弗炉中 以 $0.5{ }^{\circ} \mathrm{C} / \mathrm{min}$ 的速率, 升温至 $450{ }^{\circ} \mathrm{C}$, 恒温 $4 \mathrm{~h}$, 制 得 $\mathrm{TiO}_{2}$ 纳米纤维。

$\mathrm{Bi} / \mathrm{Bi}_{2} \mathrm{MoO}_{6} / \mathrm{TiO}_{2}$ 复合纳米纤维的制备: 取 $2.5 \mathrm{mmol} \mathrm{Bi}\left(\mathrm{NO}_{3}\right)_{3} \cdot 5 \mathrm{H}_{2} \mathrm{O}$ 和 $1 \mathrm{mmol} \mathrm{Na} \mathrm{MoO}_{4} \cdot 2 \mathrm{H}_{2} \mathrm{O}$ 分别溶于 $15 \mathrm{~mL}$ 乙二醇, 将二者混合后搅拌 $30 \mathrm{~min}$, 再加入 $0.308 \mathrm{~g} \mathrm{C}_{6} \mathrm{H}_{12} \mathrm{O}_{6}$, 继续搅拌 $30 \mathrm{~min}$, 加入 $10 \mathrm{mg}$ $\mathrm{TiO}_{2}$ 纳米纤维, 转移至 $50 \mathrm{~mL}$ 高压釜内。 $180{ }^{\circ} \mathrm{C}$ 反 应 $24 \mathrm{~h}$, 自然冷却至室温, 分别用去离子水和乙醇 洗涤纤维状产物 3 次。 $60{ }^{\circ} \mathrm{C}$ 真空干燥 $24 \mathrm{~h}$, 制得 $\mathrm{Bi} / \mathrm{Bi}_{2} \mathrm{MoO}_{6} / \mathrm{TiO}_{2}$ 复合纳米纤维, 标记为 $\mathrm{Bi} / \mathrm{BM} / \mathrm{TO}$ 。

保持上述反应条件, 不加入 $\mathrm{C}_{6} \mathrm{H}_{12} \mathrm{O}_{6}$, 制得 $\mathrm{Bi}_{2} \mathrm{MoO}_{6} / \mathrm{TiO}_{2}$ 复合纳米纤维, 标记为 $\mathrm{BM} / \mathrm{TO}$ 。

\section{3 光催化评价}

取 $20 \mathrm{mg}$ 催化剂加入 $100 \mathrm{~mL}(10 \mathrm{mg} / \mathrm{L})$ 的 $\mathrm{RhB}$ (或 4-CP)溶液中, 置于光催化反应仪中, 以 $300 \mathrm{~W}$ 氙灯 (pls-sxe300c) 为光源, 并放置滤波片, 仅使波长 大于 $400 \mathrm{~nm}$ 的光通过。反应溶液先避光搅拌 $30 \mathrm{~min}$, 然后进行光催化实验。反应过程中, 通冷却水使反 应体系保持恒温, 每隔 $10 \mathrm{~min}$ 取样 $5 \mathrm{~mL}$, 用紫外-可 见分光度计测其吸光度。

在电化学工作站上进行光电测试。采用三电极 体系, 以材料样品制得的电极为工作电极, 在室温下, 氙灯为外照光源, Pt 丝为对电极, 饱和甘录电极为 参比电极, $0.1 \mathrm{~mol} / \mathrm{L}$ 的 $\mathrm{Na}_{2} \mathrm{SO}_{4}$ 溶液为支持电解液。 光源开关间隔为 $50 \mathrm{~s}$ 。 


\section{2 结果和讨论}

\section{1 样品物相分析}

图 1 为不同样品的 XRD 图谱, 由图可见, 样品 在 $2 \theta=25.5^{\circ} 、 37.9^{\circ} 、 48.12^{\circ} 、 54.2^{\circ} 、 55.0^{\circ}$ 和 $62.8^{\circ}$ 处产生 6 个明显衍射峰, 分别与锐铁矿相 $\mathrm{TiO}_{2}$ 的 (101)、(004)、(200)、(105)、(211)和(204)晶面相匹配, 表明所制备的 $\mathrm{TiO}_{2}$ 纳米纤维为锐钛矿相。经溶剂热 反应后, $\mathrm{TiO}_{2}$ 的晶体结构完好保留, 同时在 $2 \theta$ $=28.3^{\circ} 、 32.5^{\circ} 、 33.1^{\circ} 、 36.1^{\circ} 、 46.8^{\circ} 、 47.2^{\circ} 、 55.5^{\circ} 、$ $56.3^{\circ}$ 和 $58.4^{\circ}$ 等处出现多个衍射峰, 经与标准卡片 (JCPDC 72-1524) 相比对, 分别与正交相 $\mathrm{Bi}_{2} \mathrm{MoO}_{6}$ 的 (131)、(002)、(060)、(151)、(202)、(212)、(331)、 (191)和(262)晶面衍射峰相吻合, 表明有新物种 $\mathrm{Bi}_{2} \mathrm{MoO}_{6}$ 生成。当反应体系中加入一定量的还原剂 $\left(\mathrm{C}_{6} \mathrm{H}_{12} \mathrm{O}_{6}\right)$ 时, 样品在 $2 \theta=27.3^{\circ} 、 37.1^{\circ}$ 和 $39.7^{\circ}$ 处又出 现 3 个新衍射峰(见插图), 与标准卡片(JCPDC 85-1329) 相比对，归属于金属 Bi 的(012)、(104)、(110)晶面衍

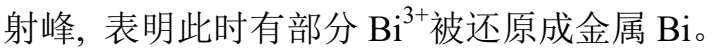

\section{2 样品形貌和结构分析}

图 2 为不同样品的 SEM 照片。由图 2(a)可见, $\mathrm{TiO}_{2}$ 纳米纤维粗细较均匀, 长径比大, 分散性良好, 表面光滑, 无其他物种附着, 直径为 250 300 $\mathrm{nm}$ 。 经溶剂热反应后, 如图 2(b)所示, 纤维表面不再光滑, 有大量 $\mathrm{Bi}_{2} \mathrm{MoO}_{6}$ 纳米片生成, 这些纳米片表面平滑, 分布均匀, 大小相近, 厚度在 10 15 $\mathrm{nm}$ 之间。当反 应体系中加入一定量 $\mathrm{C}_{6} \mathrm{H}_{12} \mathrm{O}_{6}$ 后，由图 2(c) 可见，纳 米片增大、加厚, 且表面不再平滑, 沉积有许多纳米 颗粒。图 2(d)为样品 $\mathrm{Bi} / \mathrm{BM} / \mathrm{TO}$ 的 $\mathrm{EDS}$ 能谱图, 由 图可见，该样品由 $\mathrm{Bi} 、 \mathrm{Mo} 、 \mathrm{Ti}$ 和 $\mathrm{O}$ 四种元素组成 $(\mathrm{C}$ 和 $\mathrm{Pd}$ 元素为导电胶和喷镀所致)。

图 3(a,b)为样品 BM/TO 的 TEM 和 HRTEM 照片。

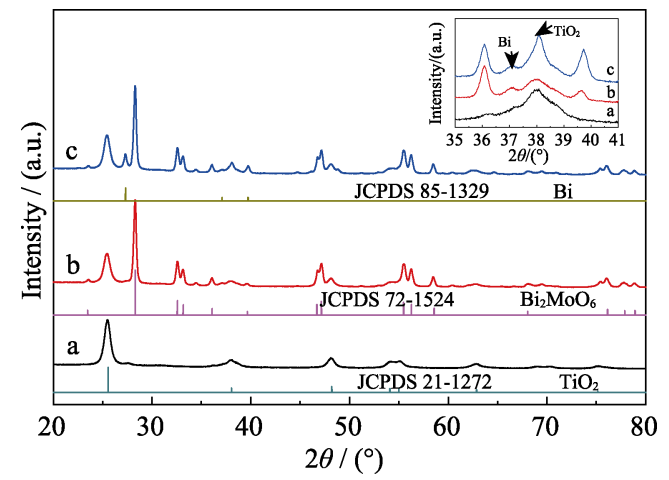

图 1 不同样品的 XRD 图谱

Fig. 1 XRD patterns of different samples a: $\mathrm{TiO}_{2} ;$ b: BM/TO; c: Bi/BM/TO
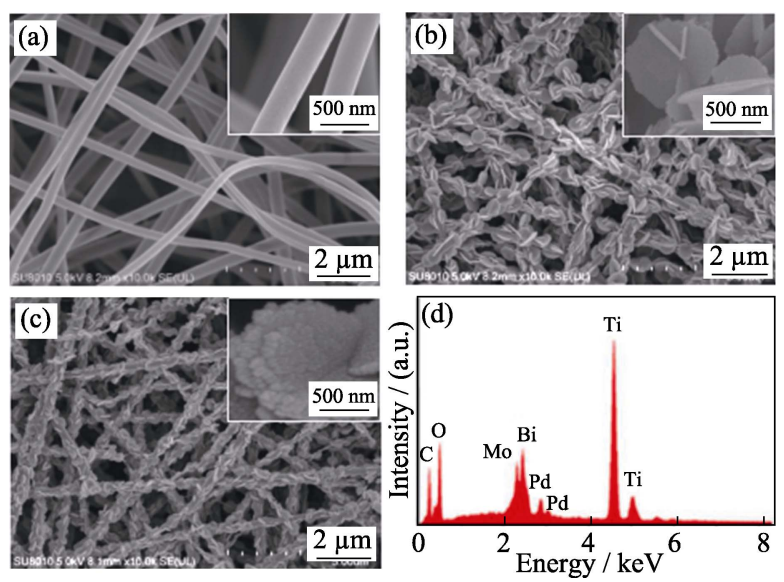

图 $2 \mathrm{TiO}_{2}(\mathrm{a}) 、 \mathrm{BM} / \mathrm{TO}(\mathrm{b})$ 和 $\mathrm{Bi} / \mathrm{BM} / \mathrm{TO}(\mathrm{c})$ 的 $\mathrm{SEM}$ 照片和样 品 $\mathrm{Bi} / \mathrm{BM} / \mathrm{TO}$ 的 EDS 能谱图(d)

Fig. 2 SEM images of $\mathrm{TiO}_{2}(\mathrm{a}), \mathrm{BM} / \mathrm{TO}(\mathrm{b}), \mathrm{Bi} / \mathrm{BM} / \mathrm{TO}$ (c) and EDS pattern of sample $\mathrm{Bi} / \mathrm{BM} / \mathrm{TO}(\mathrm{d})$

如图所示, $\mathrm{TiO}_{2}$ 纳米纤维表面构筑大量纳米片, 通过 HRTEM 清晰观察到两组不同晶格间距的衍射条纹, 其中 $0.351 \mathrm{~nm}$ 的晶格条纹对应于锐铁矿相 $\mathrm{TiO}_{2}$ 的 (101) 晶面, 而 $0.328 \mathrm{~nm}$ 的晶格条纹归属于 $\mathrm{Bi}_{2} \mathrm{MoO}_{4}$ 的(131)晶面。图 3(c $\sim$ e) 为样品 $\mathrm{Bi} / \mathrm{BM} / \mathrm{TO}$ 的 TEM 和 HRTEM 照片。由图 3(c)可见, 样品 $\mathrm{Bi} / \mathrm{BM} / \mathrm{TO}$ 表面 构筑的纳米片与样品 $\mathrm{BM} / \mathrm{TO}$ 相比增大变厚, 这与 SEM 结果一致。通过 HRTEM 观察到纳米片表面沉 积有球状纳米颗粒, 这些纳米颗粒分散性好, 大小 相近，粒径在 10 15 nm 之间(见图 3(d))。由图 3(e)同 样观察到 $\mathrm{Bi}_{2} \mathrm{MoO}_{4}$ 纳米片(131) 晶面 $0.328 \mathrm{~nm}$ 的晶 格条纹。图 3(f)是球状纳米颗粒的 HRTEM 照片, $0.270 \mathrm{~nm}$ 的晶格条纹与金属 $\mathrm{Bi}(012)$ 晶面相匹配, 表明沉积在 $\mathrm{Bi}_{2} \mathrm{MoO}_{6}$ 纳米片上的球状颗粒为金属 $\mathrm{Bi}$, 与 XRD 分析结果相符。

\section{3 光电子能谱分析}

图 4 为样品 $\mathrm{Bi} / \mathrm{BM} / \mathrm{TO}$ 的 $\mathrm{X}$ 射线光电子能谱 (XPS) 图谱。由样品的全谱图 4(a)可知, Bi/BM/TO 由 $\mathrm{Bi} 、 \mathrm{Mo} 、 \mathrm{Ti}$ 和 $\mathrm{O}$ 等四种元素构成 (其中 $\mathrm{C}$ 元素来 自碳污染)。图 4(b e ) 为样品的高分辨 XPS 能谱图。 如图 4(b) 所示, 经分峰拟合后, Bi4f 由高低两组谱峰 构成, 其中结合能位于 159.0 和 $164.4 \mathrm{eV}$ 较强的一 组谱峰, 对应于 $\mathrm{Bi}^{3+}$ 离子 $\mathrm{Bi}_{4} \mathrm{f}_{7 / 2}$ 和 $\mathrm{Bi}^{4} \mathrm{f}_{5 / 2}$ 的自旋-轨 道分裂峰, 而结合能位于 157.2 和 $162.5 \mathrm{eV}$ 较弱的 一组谱峰，对应于金属 $\mathrm{Bi}$ 的 $\mathrm{Bi}_{4} \mathrm{f}_{7 / 2}$ 和 $\mathrm{Bi} \mathrm{ff}_{5 / 2}$ 自旋轨道分裂峰 ${ }^{[23]}$, 由此证明样品中有金属 $\mathrm{Bi}$ 生成。由 图 4(c)可见, 结合能位于 232.5 和 $235.6 \mathrm{eV}$ 处的二个峰, 对应于 $M o 3 d_{5 / 2}$ 和 $M o 3 d_{3 / 2}$ 的自旋-轨道分裂峰, 说明 $\mathrm{Mo}$ 是以 +6 价存在 ${ }^{[24]}$ 。结合能位于 458.6 和 $464.4 \mathrm{eV}$ 处的二个谱峰归属于 $\mathrm{Ti} 2 \mathrm{p}_{3 / 2}$ 和 $\mathrm{Ti} 2 \mathrm{p}_{1 / 2}$ 自旋-轨道分裂 

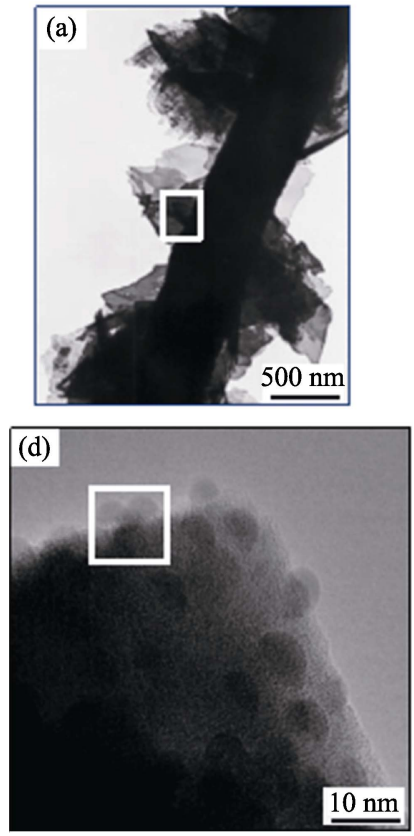
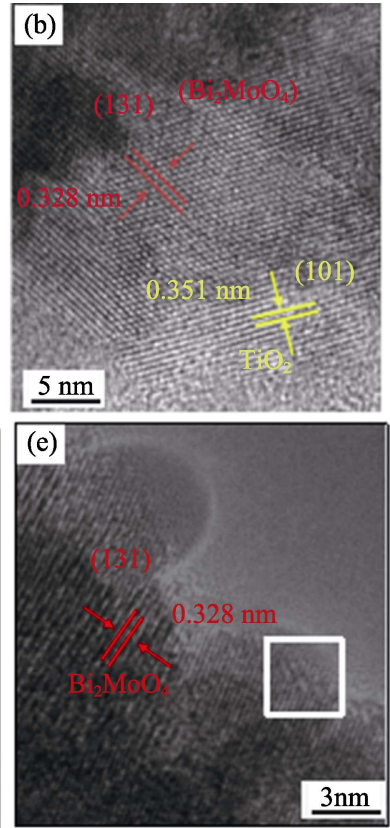
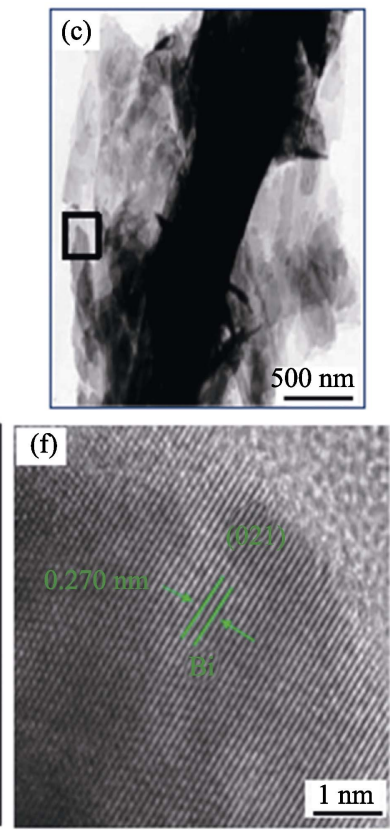

图 3 样品 $\mathrm{BM} / \mathrm{TO}(\mathrm{a}, \mathrm{b})$ 和 $\mathrm{Bi} / \mathrm{BM} / \mathrm{TO}(\mathrm{c} \sim \mathrm{f})$ 的 TEM 和 HRTEM 照片

Fig. 3 TEM and HRTEM images of sample $\mathrm{BM} / \mathrm{TO}(\mathrm{a}, \mathrm{b})$ and $\mathrm{Bi} / \mathrm{BM} / \mathrm{TO}(\mathrm{c}-\mathrm{f})$
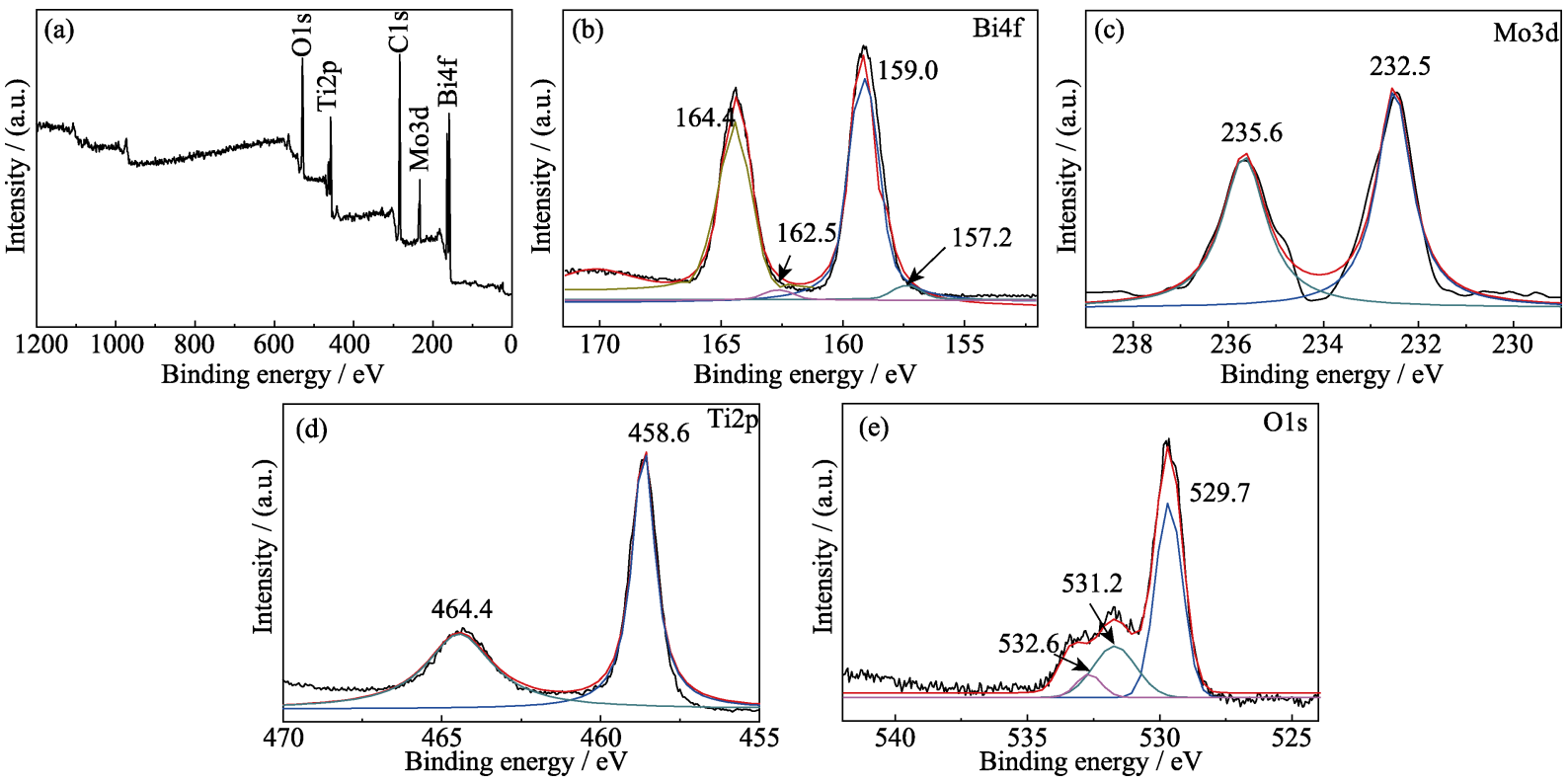

图 4 样品 $\mathrm{Bi} / \mathrm{BM} / \mathrm{TO}$ 的 XPS 谱图

Fig. 4 XPS spectra of sample $\mathrm{Bi} / \mathrm{BM} / \mathrm{TO}$

(a) Survey scan; (b) Bi4f; (c) Mo3d; (d) Ti2p; (e) O1s

峰，表明样品中 Ti 仍然以 +4 价形态存在(图 4(d)) ${ }^{[25]}$ 。 $\mathrm{O} 1 \mathrm{~s}$ 在 529.7、531.2 和 $532.6 \mathrm{eV}$ 处形成的三个谱峰 (图 4(e)), 分别对应于 $\mathrm{Bi}_{2} \mathrm{MoO}_{6}$ 中 $\left[\mathrm{Bi}_{2} \mathrm{O}_{2}\right]^{2+}$ 和 $\left[\mathrm{MoO}_{4}\right]^{2-}$ 层的晶格氧 $\left(\mathrm{O}_{\text {latt }}\right)$ 及表面吸附氧 $\left(\mathrm{O}_{\mathrm{ads}}\right)^{[26]}$ 。

\section{4 样品的光电性能分析}

图 5 为不同样品的紫外-可见漫反射光谱, 由图 可见, 锐钛矿相 $\mathrm{TiO}_{2}$ 纳米纤维仅在 $387.5 \mathrm{~nm}$ 以内的 紫外光区有强烈吸收; 与 $\mathrm{Bi}_{2} \mathrm{MoO}_{6}$ 复合后, 由于 $\mathrm{Bi}_{2} \mathrm{MoO}_{6}$ 的窄带隙特征 ${ }^{[27]}$, 使样品 $\mathrm{BM} / \mathrm{TO}$ 的吸收
带边发生红移。而样品 $\mathrm{Bi} / \mathrm{BM} / \mathrm{TO}$ 的吸收带边红移 更加明显, 且在 $450 \mathrm{~nm}$ 后的可见光区域产生较强吸 收, 主要是由金属 Bi 的 SPR 效应所致 ${ }^{[28-30]}$ 。另外, 根据 $(a h v)^{2}$ 与吸收光能量 $(h v)$ 的关系, 可以计算出以 上样品的表观带隙值分别为 3.20、2.95 和 $2.62 \mathrm{eV}$ 。

图 6 为不同样品在 300 550 nm 范围内的 PL 光 谱图, 由图可见, $\mathrm{TiO}_{2}$ 纳米纤维的荧光强度最大, 表明 其光生电子-空穴易复合。 $\mathrm{Bi}_{2} \mathrm{MoO}_{6}$ 与 $\mathrm{TiO}_{2}$ 形成了异质 结, 光生电子-空穴得到较好分离, 使样品 $\mathrm{BM} / \mathrm{TO}$ 


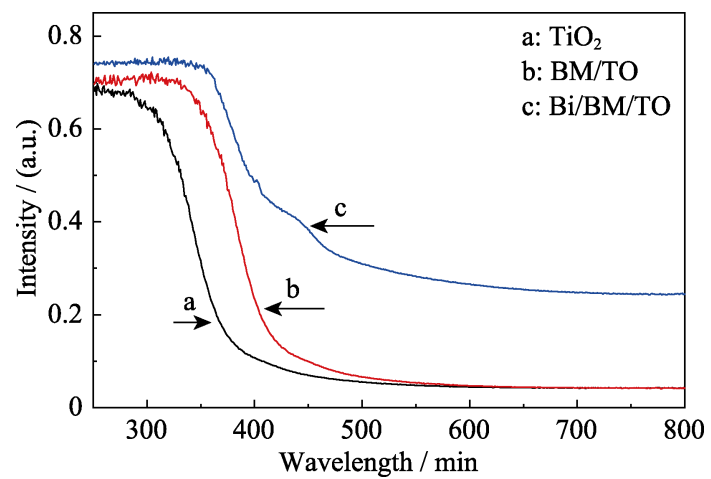

图 5 不同样品的 UV-Vis DRS 谱图

Fig. 5 UV-Vis diffuses reflectance spectra of different samples

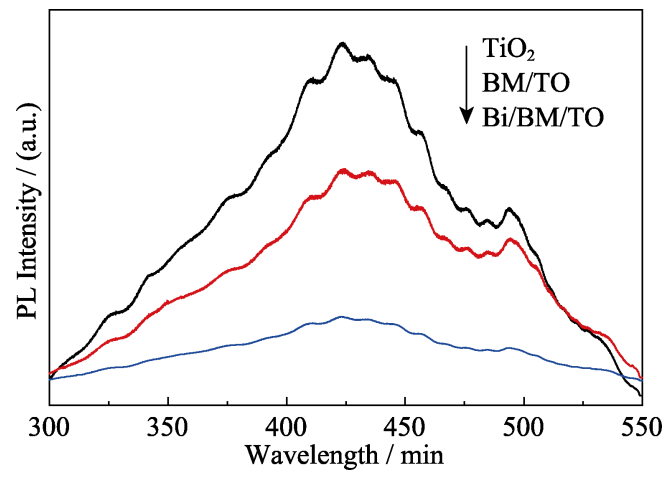

图 6 不同样品的苂光 $(\mathrm{PL})$ 光谱图

Fig. 6 PL spectra of different samples

的 PL 强度减弱。而样品 Bi/BM/TO 的 PL 强度进一 步减弱, 是因为金属 $\mathrm{Bi}$ 作为电子捕获陷荫 ${ }^{[29]}$, 接收来 自 $\mathrm{Bi}_{2} \mathrm{MoO}_{6}$ 的光生电子, 抑制了光生电子和空穴的 简单复合。

图 7 为不同样品的瞬态光电流响应图。 $\mathrm{TiO}_{2}$ 纳 米纤维只检测到微弱光电流, 表明 $\mathrm{TiO}_{2}$ 不能被可见 光激发产生光生载流子。与 $\mathrm{Bi}_{2} \mathrm{MoO}_{6}$ 复合形成异质 结及金属 $\mathrm{Bi}$ 的 $\mathrm{SPR}$ 效应 ${ }^{[31]}$, 使样品 $\mathrm{BM} / \mathrm{TO}$ 和 $\mathrm{Bi} / \mathrm{BM} / \mathrm{TO}$ 在可见光激发下均产生了较强的光电流。

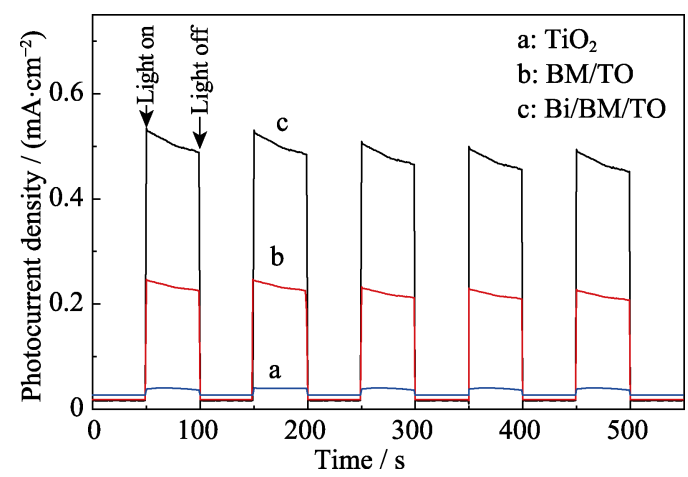

图 7 不同样品的瞬态光电流响应图

Fig. 7 Photocurrent of different samples

\section{5 光催化活性评价及机理}

首先，选择化学稳定性较好的 RhB 作为有机污 染物来评价样品的光催化性能。如图 8(a)所示, 在 无光照和不加入催化剂情况下, $\mathrm{RhB}$ 均没有变化。 $\mathrm{TiO}_{2}$ 纳米纤维为催化剂, 可见光照 $50 \mathrm{~min}, \mathrm{RhB}$ 的 降解率不到 $5 \%$, 分析认为这主要是 $\mathrm{TiO}_{2}$ 纳米纤维 的吸附引起的。BM/TO 为催化剂时, 可见光照 $50 \mathrm{~min}$, $\mathrm{RhB}$ 的降解率为 $46.7 \%$ 。而以 $\mathrm{Bi} / \mathrm{BM} / \mathrm{TO}$ 为催化剂时, $\mathrm{RhB}$ 的降解率提高到 $95.8 \%$ 。如图 8(b)所示, 对样 品光催化降解 $\mathrm{RhB}$ 动力学进行了研究, 该催化降解 过程符合一级反应, 根据 $\ln \left(C_{0} / C\right)$ 对时间 $t$ 作图, 求 得样品 $\mathrm{BM} / \mathrm{TO}$ 和 $\mathrm{Bi} / \mathrm{BM} / \mathrm{TO}$ 光催化表观反应速率常 数分别为 0.0122 和 $0.0632 \mathrm{~min}^{-1}$, 而 $\mathrm{TiO}_{2}$ 纳米纤维 的表观反应速率常数近似为 0 。

然后, 以生物难降解的 4-CP 为有机污染物, 进 一步研究了样品的可见光催化性能。如图 9(a)所示。 在可见光照射下, $\mathrm{TiO}_{2}$ 纳米纤维仅有少量吸附，仍 不具有光催化降解能力。可见光照 $180 \mathrm{~min}$, 样品 $\mathrm{BM} / \mathrm{TO}$ 和 $\mathrm{Bi} / \mathrm{BM} / \mathrm{TO}$ 对 4-CP 的降解率分别为 $35.7 \%$ 和 $68.8 \%$ 。图 9(b)为 $\mathrm{TiO}_{2}$ 纳米纤维及样品 $\mathrm{BM} / \mathrm{TO}$
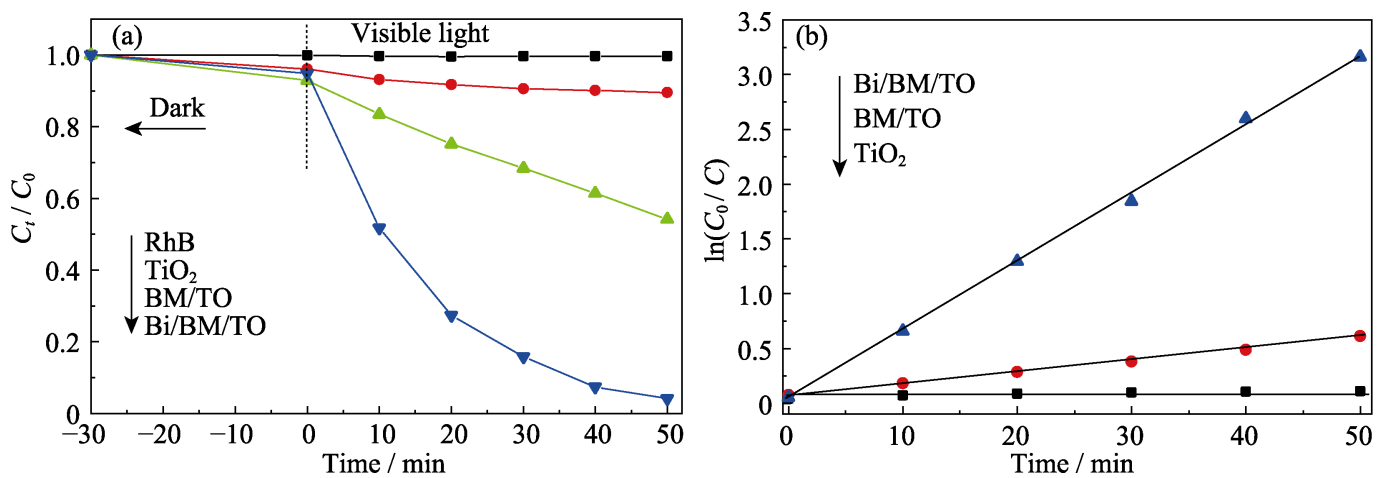

图 8 不同样品可见光催化降解 $\mathrm{RhB}(\mathrm{a})$ 和一级动力学(b)曲线

Fig. 8 Degradation curves of RhB by different samples under visible light irradiation (a) and corresponding first-order kinetics (b) 

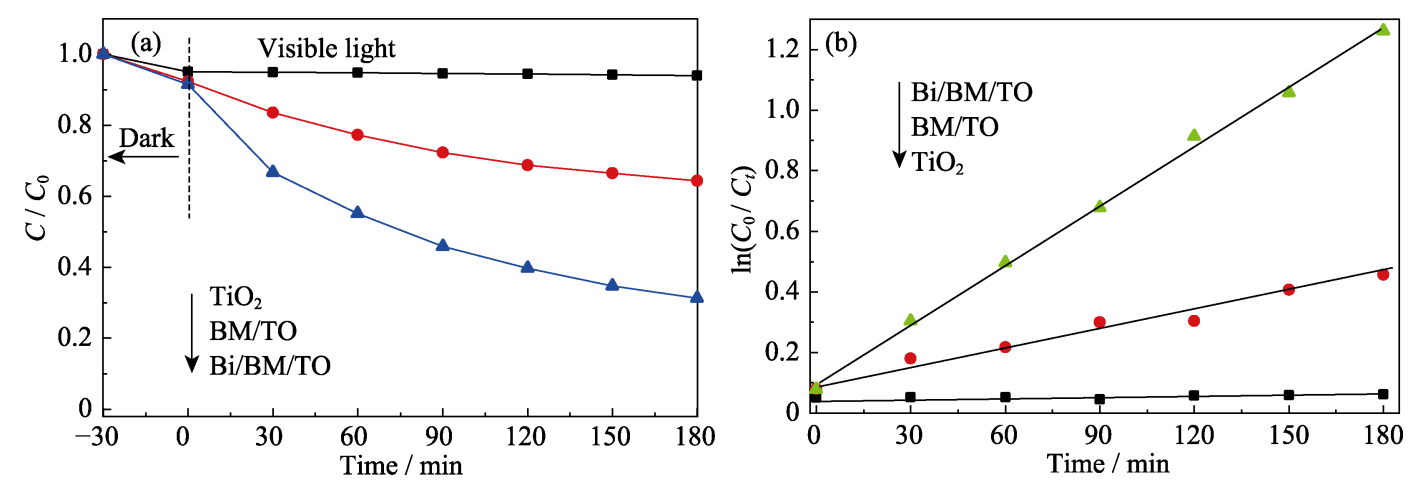

图 9 不同样品可见光催化降解 4-CP(a)和一级动力学(b)曲线

Fig. 9 Degradation curves of 4-CP by different samples under visible light irradiation (a) and corresponding first-order kinetics (b)

和 $\mathrm{Bi} / \mathrm{BM} / \mathrm{TO}$ 的一级动力学曲线, 样品 $\mathrm{BM} / \mathrm{TO}$ 和 $\mathrm{Bi} / \mathrm{BM} / \mathrm{TO}$ 光催化表观反应速率常数分别为 0.0025 和 $0.0070 \mathrm{~min}^{-1}$ 。

通过 $\mathrm{RhB}$ 光催化降解循环实验评价样品的光 催化稳定性。如图 10 所示, 样品 $\mathrm{Bi} / \mathrm{BM} / \mathrm{TO}$ 经 5 次 循环，对 RhB 的降解率仍保持在 $92 \%$ 以上，表明该 复合纳米纤维材料具有较高的稳定性和可重复使 用性，有望成为工业废水净化中的一种新型复合光 催化材料。

基于以上实验结果和理论分析, $\mathrm{Bi} / \mathrm{Bi}_{2} \mathrm{MoO}_{6} / \mathrm{TiO}_{2}$ 光催化活性及增强机理如示意图 1 所示。首先, 带隙 较窄的 $\mathrm{Bi}_{2} \mathrm{MoO}_{6}$ 与 $\mathrm{TiO}_{2}$ 形成异质结, $\mathrm{Bi}_{2} \mathrm{MoO}_{6}$ 的导带 电位 $(-0.32 \mathrm{eV})^{[27]}$ 更负于 $\mathrm{TiO}_{2}$ 的导带电位 $(-0.23 \mathrm{eV})^{[32]}$, $\mathrm{Bi}_{2} \mathrm{MoO}_{6}$ 导带上光生电子穿过异质结迁移到 $\mathrm{TiO}_{2}$ 导 带, 使光生电子与空穴得到较好分离, 延长了光生载 流子寿命，提高了光催化活性。其次，是金属 $\mathrm{Bi}$ 的 $\mathrm{SPR}$ 效应, $\mathrm{Bi}$ 作为电子捕获陷阱，接收 $\mathrm{Bi}_{2} \mathrm{MoO}_{6}$ 导带 上的电子 ${ }^{[27]}$, 有效抑制了光生电子和空穴的简单复 合，同时金属 $\mathrm{Bi}$ 在可见光区产生较强的吸收，能够激 发出更多的光生载流子, 有效提高光催化活性和太 阳光利用率 ${ }^{[33]}$ 。第三，是 SPR 效应与异质结的协同 作用。金属 $\mathrm{Bi}$ 与半导体 $\mathrm{Bi}_{2} \mathrm{MoO}_{6}$ 相接触, 在二者界 面处 $\mathrm{Bi}_{2} \mathrm{MoO}_{6}$ 的能带发生弯曲, 形成一个高势能的 肖特基垒，电子必须高于这一势垒的能量才能越过势 垒流向金属 $\mathrm{Bi}$ 产生局部电磁场, 这个局部电磁场作 用于 $\mathrm{Bi}_{2} \mathrm{MoO}_{6} / \mathrm{TiO}_{2}$ 异质结内部电场，降低 $\mathrm{Bi}_{2} \mathrm{MoO}_{6}$ 一侧的能量势垒, 使更多的光生电子和空穴得到有 效分离，增强了样品的光催化活性 ${ }^{[27,29] 。}$

迁移到催化剂表面的电子和空穴与吸附在催化 剂表面的 $\mathrm{O}_{2}$ 和 $\mathrm{H}_{2} \mathrm{O}$ 等反应生成超氧自由基 $\left(\cdot \mathrm{O}_{2}{ }^{-}\right)$和 羟基自由基 $(\cdot \mathrm{OH})$ 等活性物种, 均具有很强的氧化 能力, 可以将溶液中 $\mathrm{RhB}$ 或 4-CP 最终氧化分解为 $\mathrm{CO}_{2}$ 和 $\mathrm{H}_{2} \mathrm{O}$ 等无机小分子, 达到光催化有机污染物 的目的。

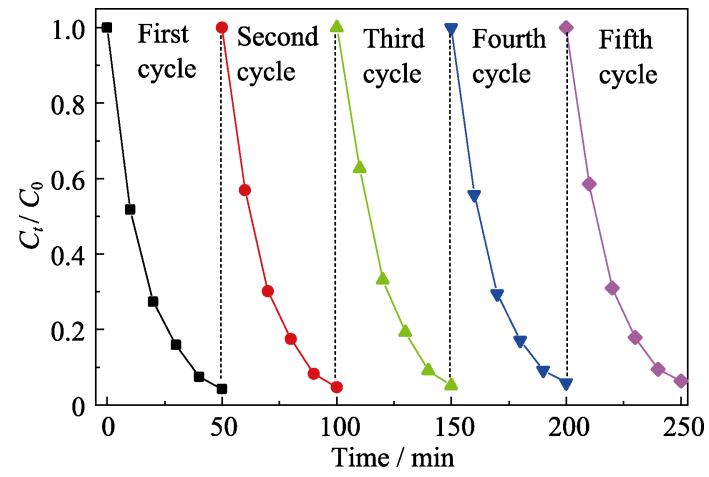

图 10 可见光下 $\mathrm{Bi} / \mathrm{BM} / \mathrm{TO}$ 对 $\mathrm{RhB}$ 光催化的循环实验

Fig. 10 Cycling runs of the photocatalytic degradation of $\mathrm{RhB}$ over $\mathrm{Bi} / \mathrm{BM} / \mathrm{TO}$ under visible irradiation

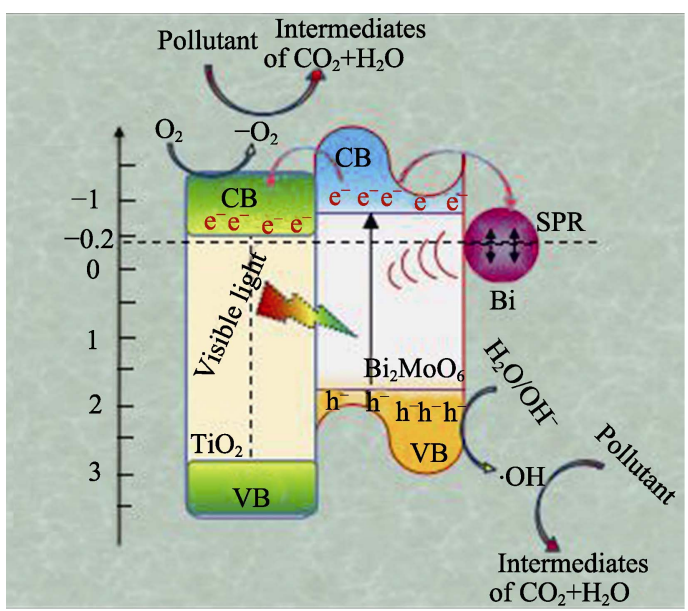

示意图 1 样品 $\mathrm{Bi} / \mathrm{BM} / \mathrm{TO}$ 可见光催化降解及能带结构图 Scheme 1 Proposed photocatalytic mechanism for photodegradation of organic pollutants over $\mathrm{Bi} / \mathrm{BM} / \mathrm{TO}$

\section{3 结论}

以 $\mathrm{TiO}_{2}$ 纳米纤维为基质，葡萄糖作还原剂，采 用简单的一步溶剂热法成功制备了 $\mathrm{Bi} / \mathrm{Bi}_{2} \mathrm{MoO}_{6} / \mathrm{TiO}_{2}$ 复合纳米纤维光催化材料。在水热过程中, 部分 $\mathrm{Bi}^{3+}$ 被葡萄糖还原成金属 $\mathrm{Bi}$ 沉积在 $\mathrm{Bi}_{2} \mathrm{MoO}_{6}$ 纳米片上, 同时构筑到 $\mathrm{TiO}_{2}$ 纳米纤维表面。金属 $\mathrm{Bi}$ 的引入对 
$\mathrm{Bi}_{2} \mathrm{MoO}_{6}$ 纳米片的大小、厚度以及光电性能都产生 了影响, 有效提高了样品的光催化活性和稳定性。 本研究对于设计新型高效可见光催化材料, 具有一 定参考价值。

\section{参考文献:}

[1] CHEN C C, MA W H, ZHAO J C. Semiconductor-mediated photodegradation of pollutants under visible-light irradiation. Chem. Soc. Rev., 2010, 39(11): 4206-4219.

[2] LANG X J, CHEN X D, ZHAO J. Heterogeneous visible light photocatalysis for selective organic transformations. Chem. Soc. Rev., 2014, 43(1): 473-486.

[3] CAO T P, LI Y J, SHAO C L, et al. A facile in situ hydrothermal method to $\mathrm{SrTiO}_{3} / \mathrm{TiO}_{2}$ nanofiber heterostructures with high photocatalytic activity. Langmuir, 2011, 27(6): 2946-2952.

[4] TONG H, OUYANG S X, BI Y P, et al. Nano-photocatalytic materials: possibilities and challenges. Adv. Mater., 2012, 24(2): 229-251.

[5] LI J, YU Y, ZHANG L Z. Bismuth oxyhalide nanomaterials: layered structures meet photocatalysis. Nanoscale, 2014, 6(15): 8473-8488.

[6] TIAN J, HAO P, WEI N, et al. 3D $\mathrm{Bi}_{2} \mathrm{MoO}_{6}$ nanosheet $/ \mathrm{TiO}_{2}$ nanobelt heterostructure: enhanced photocatalytic activities and photoelectochemistry performance. ACS Catal., 2015, 5(8): 4530-4536.

[7] LI X X, FANG S M, GE L, et al. Synthesis of flower-like $\mathrm{Ag} / \mathrm{AgCl}-\mathrm{Bi}_{2} \mathrm{MoO}_{6}$ plasmonic photocatalysts with enhanced visible-light photocatalytic performance. Appl. Catal. B: Environ., 2015, 176-177: 62-69.

[8] WU M H, WANG Y X, XU Y, et al. Self-supported $\mathrm{Bi}_{2} \mathrm{MoO}_{6}$ nanowall for photoelectrochemical water splitting. ACS Appl. Mater. Interfaces, 2017, 9(28): 23647-23653.

[9] SUN Y Y, WANG W Z, SUN S M, et al. A general synthesis strategy for one-dimensional $\mathrm{Bi}_{2} \mathrm{MO}_{6}(\mathrm{M}=\mathrm{Mo}, \mathrm{W})$ photocatalysts using an electrospinning method. CrystEngComm, 2013, 15(39): 7959-7964.

[10] DAI W L, YU J J, XU H, et al. Synthesis of hierarchical flower-like $\mathrm{Bi}_{2} \mathrm{MoO}_{6}$ microspheres as efficient photocatalyst for photoreduction of $\mathrm{CO}_{2}$ into solar fuels under visible light. CrystEngComm, 2016, 18(19): 3472-3480.

[11] ZHAO J, LU Q F, WANG C Q, et al. One-dimensional $\mathrm{Bi}_{2} \mathrm{MoO}_{6}$ nanotubes: controllable synthesis by electrospinning and enhanced simulated sunlight photocatalytic degradation performances. $J$. Nanopart. Res., 2015, 17: 189-199.

[12] YU C L, WU Z, LIU R Y, et al. Novel fluorinated $\mathrm{Bi}_{2} \mathrm{MoO}_{6}$ nanocrystals for efficient photocatalytic removal of water organic pollutants under different light source illumination. Appl. Catal. B: Environ., 2017, 209: 1-11.

[13] AWAZU K, FUJIMAKI M, ROCKSTUHL C, et al. A plasmonic photocatalyst ponsisting of silver nanoparticles embedded in titanium dioxide. J. Am. Chem. Soc., 2008, 130(5): 1676-1680.

[14] MORI K, VERMA P, HAYASHI R, et al. Color-controlled Ag nanoparticles and nanorods within confined mesopores: microwave-assisted rapid synthesis and application in plasmonic catalysis under visible-light irradiation. Chem-A. Eur. J., 2015, 21(33): 11885-11893.

[15] YANG J, WANG X H, CHEN Y M, et al. Enhanced photocatalytic activities of visible-light driven green synthesis in water and environmental remediation on $\mathrm{Au} / \mathrm{Bi}_{2} \mathrm{WO}_{6}$ hybrid nanostructures. $R S C$ $A d v$, 2015, 5(13): 9771-9782.

[16] WU Q S, CUI Y, YANG L M, et al. Facile in-situ photocatalysis of $\mathrm{Ag} / \mathrm{Bi}_{2} \mathrm{WO}_{6}$ heterostructure with obviously enhanced perform- ance. Sep. Purif. Technol., 2015, 142: 168-175.

[17] YU C L, BAI Y, CHEN J C, et al. $\mathrm{Pt} / \mathrm{Bi}_{2} \mathrm{WO}_{6}$ composite microflowers: high visible light photocatalytic performance and easy recycle. Sep. Purif. Technol., 2015, 154: 115-122.

[18] QIN F, WANG R M, LI G F, et al. Highly efficient photocatalytic reduction of $\mathrm{Cr}(\mathrm{VI})$ by bismuth hollow nanospheres. Catalysis Communications, 2013, 42: 14-19.

[19] WANG Y W, KIM J S, KIM G H, et al. Quantum size effects in the volume plasmon excitation of bismuth nanoparticles investigated by electron energy loss spectroscopy. Appl. Phys. Lett., 2006, 88(14): 143106-143109.

[20] LIU X W, CAO H Q, YIN JIE F, et al. Generation and photocatalytic activities of $\mathrm{Bi} @ \mathrm{Bi}_{2} \mathrm{O}_{3}$ microspheres. Nano Res., 2011, 4(5): 470-482.

[21] WENG S X, CHEN B B, XIE L Y, et al. Facile in situ synthesis of a $\mathrm{Bi} / \mathrm{BiOCl}$ nanocomposite with high photocatalytic activity. J. Mater. Chem. A, 2013, 1(9): 3068-3075.

[22] GNAYEM H, SASSON Y. Nanostructured 3D sunflower-like bismuth doped $\mathrm{BiOCl}_{x} \mathrm{Br}_{1-x}$ solid dolutions with enhanced visible light photocatalytic activity as a remarkably efficient technology for water purification. J. Phys. Chem. C, 2015, 119(33): 19201-19209.

[23] YANG J, WANG X H, ZHAO X L, et al. Synthesis of uniform $\mathrm{Bi}_{2} \mathrm{WO}_{6}$-reduced graphene oxide nanocomposites with significantly enhanced photocatalytic reduction activity. J. Phys. Chem. C, 2015, 119: 3068-3078.

[24] LÜ W Z, HUANG D Z, CHEN Y M, et al. Synthesis and characterization of Mo-W co-doped $\mathrm{VO}_{2}(\mathrm{R})$ nano-powders by the microwave-assisted hydrothermal method. Ceram. Int., 2014, 40(8): 12661-12668.

[25] REN W J, AI Z H, JIA F L, et al. Low temperature preparation and visible light photocatalytic activity of mesoporous carbon-doped crystalline $\mathrm{TiO}_{2}$. Appl. Catal. B-Environ., 2007, 69(34): 138-144.

[26] ZHOU Y G, ZHANG Y F, LIN M S, et al. Monolayered $\mathrm{Bi}_{2} \mathrm{WO}_{6}$ nanosheets mimicking heterojunction interface with open surfaces for photocatalysis. Nat. Commun., 2015, 6: 8340-8347.

[27] ZHAO Z W, ZHANG W D, DONG F, et al. Bi cocatalyst/Bi ${ }_{2} \mathrm{MoO}_{6}$ microspheres nanohybrid with SPR-promoted visible-light photocatalysis. J. Phys. Chem. C, 2016, 120(22): 11889-11898.

[28] LU S Y, YU Y N, BAO S J, et al. In situ synthesis and excellent photocatalytic activity of tiny Bi decorated bismuth tungstate nanorods. RSC Advances, 2015, 5: 85500-85505.

[29] DONG F, LI Q Y, SUN Y J, et al. Noble metal-like behavior of plasmonic Bi particles as a cocatalyst deposited on $(\mathrm{BiO})_{2} \mathrm{CO}_{3}$ microspheres for efficient visible light photocatalysis. ACS Catal., 2014, 4(12): 4341-4350

[30] WANG Z, JIANG C L, HUANG R, et al. Investigation of optica and photocatalytic properties of bismuth nanospheres prepared by a facile thermolysis method. J. Phys. Chem. C, 2014, 118(2): 1155-1160.

[31] YU Y, CAO C Y, LIU H, et al. A Bi/BiOCl heterojunction photocatalyst with enhanced electron-hole separation and excellent visible light photodegrading activity. J. Mater. Chem. A , 2014, 2(6): 1677-1681.

[32] FENG H B, LI Y P, QIAN D, et al. Novel visible-light-responding $\mathrm{InVO}_{4}-\mathrm{Cu}_{2} \mathrm{O}-\mathrm{TiO}_{2}$ ternary nanoheterostructure: preparation and photocatalytic characteristics. Chinese Journal of Catalysis, 2016, 37(6): 855-862.

[33] FURUBE A, DU L C, HARA K, et al. Ultrafast plasmon-induced electron transfer from gold nanodots into $\mathrm{TiO}_{2}$ nanoparticles. $J$. Am. Chem. Soc., 2007, 129(48): 14852-14853. 